\title{
Acidic leucine-rich nuclear phosphoprotein-32A (ANP32A) association with lymph node metastasis predicts poor survival in oral squamous cell carcinoma patients
}

\author{
Bharath Kumar Velmurugan ${ }^{1, *}$, Kun-Tu Yeh ${ }^{2,3, *}$, Chien-Hung Lee ${ }^{4}$, Shu-Hui Linn ${ }^{2,3}$, \\ Mei-Chung Chin ${ }^{2}$, Shang-Lun Chiang ${ }^{1,5}$, Zhi-Hong Wang ${ }^{1}$, Chun-Hung Hua ${ }^{6}$, Ming- \\ Hsui Tsai ${ }^{6}$, Jan-Gowth Chang7 and Ying-Chin Ko ${ }^{1,8}$ \\ ${ }^{1}$ Environment-Omics-Diseases Research, China Medical University Hospital, Taichung, Taiwan \\ 2 Department of Pathology, Changhua Christian Hospital, Changhua, Taiwan \\ ${ }^{3}$ School of Medicine, Chung Shan Medical University, Taichung, Taiwan \\ ${ }^{4}$ Department of Public Health, College of Health Science, Kaohsiung Medical University, Taiwan \\ ${ }^{5}$ Department of Health Risk Management, College of Public Health, China Medical University, Taichung, Taiwan \\ ${ }^{6}$ Department of Otorhinolaryngology, China Medical University Hospital, Taichung, Taiwan \\ ${ }^{7}$ Department of Laboratory Medicine, China Medical University Hospital, Taichung, Taiwan \\ ${ }^{8}$ Graduate Institute of Clinical Medical Science, China Medical University, Taichung, Taiwan \\ * These authors have contributed equally to this work \\ Correspondence to: Ying-Chin Ko, email: ycko0406@gmail.com \\ Keywords: OSCC, Claudlin-1, ANP32A, Slug, EMT, slug, E-cadherin, vimentin \\ Received: November 15,2015 Accepted: February 16, $2016 \quad$ Published: February 24, 2016
}

ABSTRACT

Acidic leucine-rich nuclear phosphoprotein-32A (ANP32A) is a multifunctional protein aberrantly expressed in various types of cancers. However, its expression pattern and clinical significance in oral squamous cell carcinoma (OSCC) remains unclear. In this study, we immunohistochemically investigated the expression pattern of ANP32A in 259 OSCC patients and the results were correlated with clinicopathological factors using Allred, Klein and Immunoreactive scoring (IRS) system. Our data indicated that high expression of ANP32A was significantly associated with $\mathbf{N}$ stage and tumor differentiation status in OSCC patients. High ANP32A expression with N2/N3 stage had an increased mortality risk than low ANP32A expressing OSCC patients with NO/N1 stage. Functional studies revealed that knockdown of ANP32A significantly decreased the migration and invasion ability thereby concomitantly increasing E-cadherin and decreasing Slug, Claudin-1 and Vimentin expression in vitro. These results suggest that ANP32A is commonly increased in oral squamous cell carcinoma and ANP32A protein could act as a potential biomarker for prognosis assessment of oral cancer patients with lymph node metastasis.

\section{INTRODUCTION}

Oral squamous cell carcinoma (OSCC) is the sixth leading cause of cancer death in the worldwide (The agestandardized per 100,000 GLOBOCAN 2008, IARC); in Taiwan more than 6500 new cases of oral and pharyngeal cancer are diagnosed annually (7-8\% of all cancers) [1]. Despite of the improved diagnostic technique its prognosis remains poor; indeed, the 5-year survival rate remained around $50-55 \%$ over the past several decades [2-4]. Therefore, it is of important to identify the molecular markers that are associated with OSCC malignancy; which may further improve the clinical management and therapeutic development $[5,6]$.

Acidic leucine rich nuclear phosphoprotein32A (ANP32A) is nuclear phosphorproteins that are expressed in normal tissues as well as in breast, pancreas, and prostate cancers [7-9]. ANP32A (pp32), possess 
variety of cellular functions such as cell differentiation, transcription, apoptosis and cell cycle progression $[8,10-$ 12]. In pancreatic adenocarcinoma expression of ANP32A was well correlated with their differentiation status. This well differentiated tumors showed normal expression of ANP32A, whereas reduced or absent in poorly differentiated tumors [13]. Most of the studies have found that, ANP32A function as a tumor suppressor protein $[9,14]$, surprisingly increased expression was found in hepatocellular carcinoma[15], colorectal cancer[16], pancreatic tumor[17] and liver cancer [15]. However, whether the aberrant expression of ANP32A in OSCC is associated with malignancy, metastasis, or prognosis remains unknown.

In this present study we determined the expression pattern of ANP32A in oral squamous cell carcinoma and its correlation with clinicopathological features, including the overall survival of OSCC patients. Additionally, we examined the functional role of ANP32A on EMT pathway using highly invasive oral cancer cell- HSC-3, in vitro

\section{RESULTS}

\section{Expression profile of ANP32A in tumor and normal tissues of OSCC patients}

To study the prevalence of ANP32A in OSCC patients, at first we examined the expression pattern of ANP32A using immunohistochemistry on the tumor tissues and adjacent non-tumor tissues from 259 patients with OSCC (Figure 1). ANP32A was observed in both nucleus and cytoplasm of OSCC cells and in non-tumor tissues. Compared to the non-tumor tissues, the level of ANP32A staining was higher in the OSCC cells. ANP32A staining was scored as " $1+$ " if the staining intensity was lower than the staining intensity of the normal oral squamous mucosa. The staining was scored as " $2+$ " if the staining intensity in the neoplasm matched the staining intensity of the normal oral squamous mucosa. A score of " \pm " reflected a lack of ANP32A immunoreactivity compared with the staining pattern of normal oral squamous mucosa. In a total of 259 OSCC cases, ANP32A expression was negative or weak positive $( \pm)$ in 114 cases, moderately positive in 105 cases (with IHC scores of $1+$ ) and strongly positive in 40 cases (with IHC scores of 2+). To determine the antibody specificity we used endometrial cancer as a positive control and breast cancer as a negative control (Figure 1e \& 1f).

\section{Association between ANP32A and clinicopathologic factors}

The demographic and clinicopathologic characteristics for OSCC patients were shown in Table
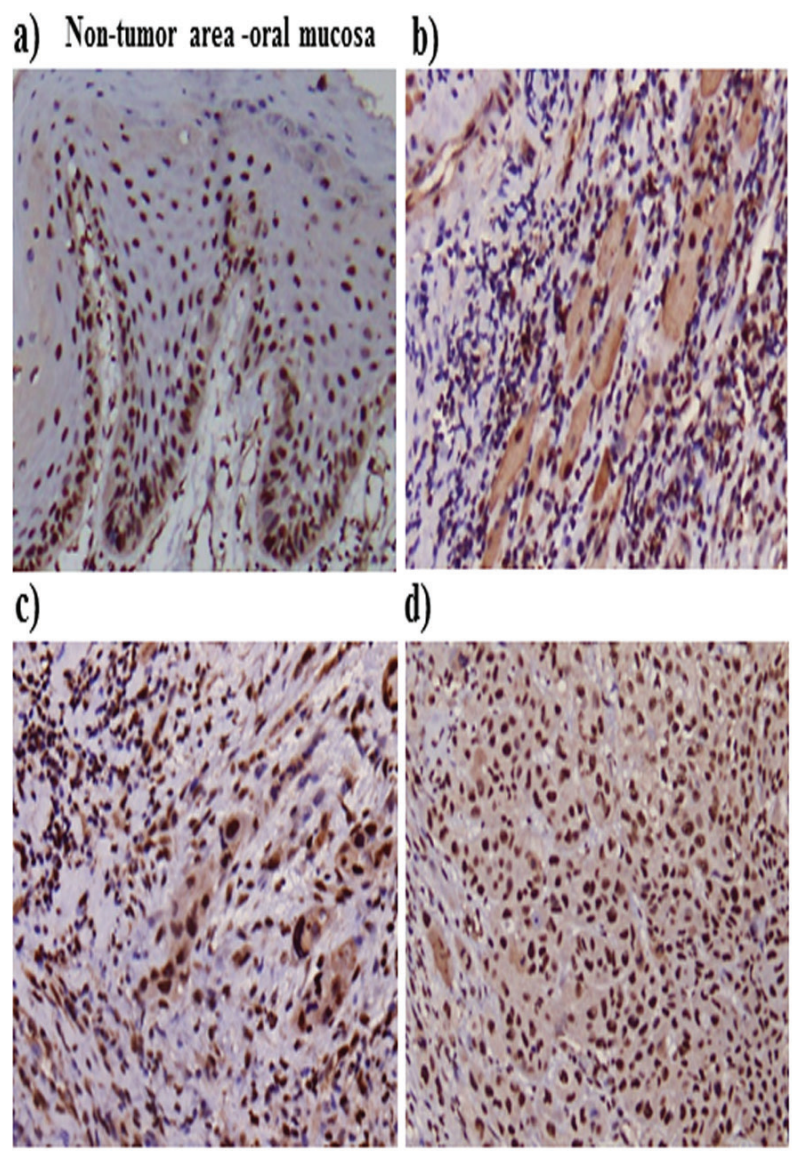

d)

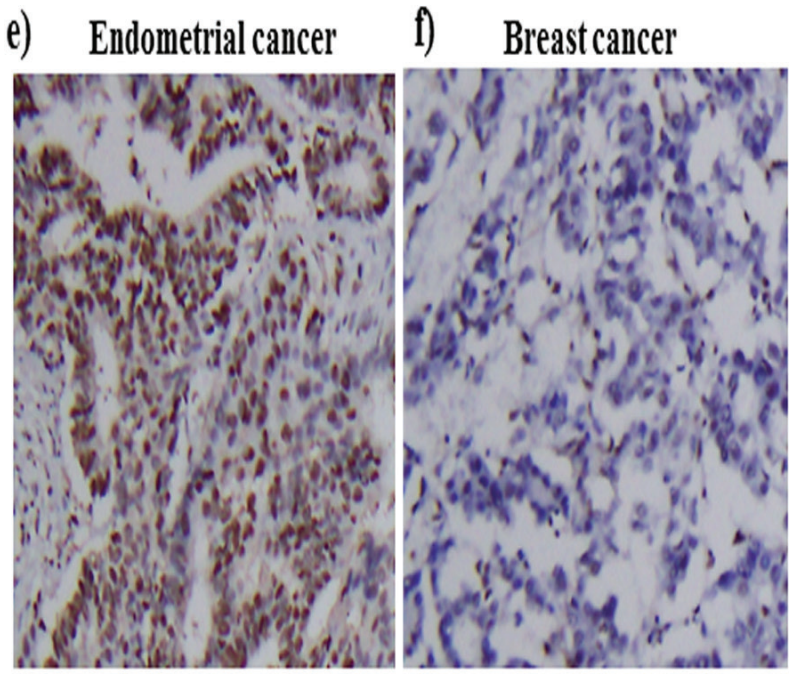

Figure 1: Higher expression of AN32Ain OSCC patients. ANP32A expression was detected in oral cancer patients and in non-tumor tissues by performing immunohistochemistry. a. ANP32A expression in the non-tumor areas of oral mucosa. b.-d. Different staining intensities of ANP32A in OSCC tissues. The scoring criteria for ANP32A immunoreactivity are described in the Materials and methods section. The cellular staining was classified using a scale of $( \pm, 1+, 2+)$ as follows: \pm weak staining b., $1+$ moderate staining c) $2+$ strong staining d..e. $\&$ f. Endometrial and breast cancer was used as a positive and negative control respectively. Representative images show the consistent expression of ANP32A.Left panel: magnification $\times 100$. Right panel: magnification $\times 400$. 
Table 1: Demographic and characteristics among oral cancer patients

\begin{tabular}{|c|c|c|}
\hline Factors & No & $\%$ \\
\hline \multicolumn{3}{|l|}{ Gender } \\
\hline Female & 13 & 5.0 \\
\hline Male & 246 & 95.0 \\
\hline \multicolumn{3}{|l|}{ Age, year } \\
\hline$\leq 49$ & \begin{tabular}{|l|}
79 \\
\end{tabular} & 30.5 \\
\hline $50-59$ & 95 & 36.7 \\
\hline $60-69$ & 57 & 22.0 \\
\hline$\geq 70$ & 28 & 10.8 \\
\hline \multicolumn{3}{|l|}{$\mathrm{T}$ (tumor size) } \\
\hline $\mathrm{I}$ & 64 & 24.7 \\
\hline II & 81 & 31.3 \\
\hline III & 22 & 8.5 \\
\hline IV & 92 & 35.5 \\
\hline \multicolumn{3}{|l|}{ N (lymph node) } \\
\hline N0 & 167 & 64.5 \\
\hline N1 & 33 & 12.7 \\
\hline $\mathrm{N} 2$ & 55 & 21.2 \\
\hline N3 & 4 & 1.5 \\
\hline \multicolumn{3}{|l|}{ M (metastasis) } \\
\hline No & 257 & 99.6 \\
\hline Yes & 1 & 0.4 \\
\hline \multicolumn{3}{|l|}{ AJCC cancer stage } \\
\hline I & 50 & 19.3 \\
\hline II & 54 & 20.9 \\
\hline III & 33 & \begin{tabular}{|l|}
12.7 \\
\end{tabular} \\
\hline IV & 122 & 47.1 \\
\hline \multicolumn{3}{|l|}{ Histological grade } \\
\hline Well & 41 & 15.8 \\
\hline Moderate & 211 & 81.5 \\
\hline Poor & 7 & 2.7 \\
\hline \multicolumn{3}{|l|}{ Clinical therapy } \\
\hline Surgery & 98 & 37.8 \\
\hline Radiotherapy & 96 & 37.1 \\
\hline Chemotherapy & 1 & \begin{tabular}{|l|l}
0.4 \\
\end{tabular} \\
\hline Chemoradiotherapy & 64 & 24.7 \\
\hline
\end{tabular}

1. A predominant proportion of oral malignant cases were male $(95 \%)$, as compared to female $(5 \%)$.Among the cancer patients, $44 \%$ and $22.7 \%$ were found to have tumor site of stage III/IV and lymph node metastasis of $\mathrm{N} 2 / \mathrm{N} 3$, respectively, and $84.2 \%$ were moderate or poor histological grade. A total of 98 OSCC patients $(37.8 \%)$ received surgery therapy and 161 patients $(62.2 \%)$ were treated with radiotherapy or/and chemotherapy.

The association between clinicopathologic factors and ANP32A expression by different IHC scoring systems in OSCC patients was presented in Table 2. Patients with a high level of ANP32A expression defined by Allred and IRS scoring systems were respectively found to have a 2.2-fold (95\% CI, 1.2-4.0) and 2.0-fold (95\% CI, 1.13.6) risk of contracting $\mathrm{N} 2 / \mathrm{N} 3$ of lymph node metastasis.
Likewise, a higher ANP32A expression was related to moderate/poor tumor differentiation in these two scoring systems (adjusted odds ratios, aOR $=2.2, P<0.05$, in both systems). No significant relationship between ANP32A expression and other clinicopathologic parameters were identified. However, using Klein scoring system no any significant association was found between ANP32A expression and pathological parameters. This results are in correlation with Fedchenko $\mathrm{N}$ et al [18] hypothesis that, each IHC marker should have an individual scoring systems. Thus we chose to use IRS and Allred scoring system to interpret and further analysis our results. 
Table 2: Clinicopathologic factors associated with ANP32A expression by different IHC scoring systems among OSCC patients

\begin{tabular}{|c|c|c|c|c|c|c|c|c|c|c|c|c|}
\hline \multirow[b]{2}{*}{ Factors } & \multicolumn{4}{|c|}{ Allred } & \multicolumn{4}{|c|}{ Klein } & \multicolumn{4}{|c|}{ IRS } \\
\hline & $\begin{array}{c}\text { Low } \\
\text { No. } \\
(n=151) \\
\end{array}$ & $\begin{array}{c}\text { High } \\
\text { No. } \\
(\mathrm{n}=108)\end{array}$ & $\mathrm{aOR}^{\mathrm{a}}$ & $(95 \% \mathrm{CI})$ & $\begin{array}{c}\text { Low } \\
\text { No. } \\
(\mathrm{n}=151\end{array}$ & $\begin{array}{c}\text { High } \\
\text { No. } \\
(\mathrm{n}=108) \\
\end{array}$ & $\mathbf{a O R}^{\mathrm{a}}$ & $(95 \%$ CI $)$ & $\begin{array}{c}\text { Low } \\
\text { No. } \\
(n=159) \\
\end{array}$ & $\begin{array}{c}\text { High } \\
\text { No. } \\
(\mathrm{n}=100) \\
\end{array}$ & $\mathbf{a O R}^{\mathrm{a}}$ & $(95 \% \mathrm{CI})$ \\
\hline \multicolumn{13}{|l|}{$\overline{\mathrm{T} \text { (tumor size) }}$} \\
\hline $\mathrm{I} / \mathrm{II}$ & 83 & 62 & 1.0 & & 126 & 19 & 1.0 & & 87 & 58 & 1.0 & \\
\hline III/IV & 68 & 46 & 0.9 & $(0.6-1.5)$ & 98 & 16 & 1.1 & $(0.6-2.4)$ & 72 & 42 & 0.9 & $(0.5-1.5)$ \\
\hline \multicolumn{13}{|l|}{ N (lymph node) } \\
\hline N0/N1 & 125 & 75 & 1.0 & & 176 & 24 & 1.0 & & 130 & 70 & 1.0 & \\
\hline $\mathrm{N} 2 / \mathrm{N} 3$ & 26 & 33 & 2.2 & $(1.2-4.0)$ & 48 & 11 & 1.8 & $(0.8-4.0)$ & 29 & 30 & 2.0 & $(1.1-3.6)$ \\
\hline \multicolumn{13}{|l|}{ M (metastasis) } \\
\hline No & 150 & 107 & 1.0 & & 222 & 35 & 1.0 & & 158 & 99 & 1.0 & \\
\hline Yes & 0 & 1 & - & ND & 1 & 0 & - & ND & 0 & 1 & - & ND \\
\hline \multicolumn{13}{|l|}{ AJCC cancer stage } \\
\hline I & 32 & 18 & 1.0 & & 46 & 4 & 1.0 & & 33 & 17 & 1.0 & \\
\hline II & 30 & 24 & 1.5 & $(0.7-3.4)$ & 46 & 8 & 2.0 & $(0.6-7.4)$ & 33 & 21 & 1.3 & $(0.6-3.0)$ \\
\hline III & 20 & 13 & 1.3 & $(0.5-3.3)$ & 28 & 5 & 2.3 & $(0.6-9.7)$ & 20 & 13 & 1.5 & $(0.6-3.8)$ \\
\hline IV & 69 & 53 & 1.5 & $(0.7-3.0)$ & 104 & 18 & 2.2 & $(0.7-6.9)$ & 73 & 49 & 1.4 & $(0.7-2.9)$ \\
\hline \multicolumn{13}{|l|}{ Histological grade } \\
\hline Well & 30 & 11 & 1.0 & & 37 & 4 & 1.0 & & 31 & 10 & 1.0 & \\
\hline Moderate/Poor & 121 & 97 & 2.2 & $(1.0-4.7)^{*}$ & 187 & 31 & 1.5 & $(0.5-4.7)$ & 128 & 90 & 2.2 & $(1.0-4.7)^{*}$ \\
\hline
\end{tabular}

${ }^{a}$ Adjusted odds ratio (aOR) was controlled for gender and age.

$* P<0.05 ; \mathrm{ND}$, not determined.

\section{ANP32A expression and survival}

The effects of clinicopathologic factor and ANP32A expression on mortality in the OSCC patient cohort were shown in Table 3. The mean follow-up time for this cohort was 5.4 year (SD, 3.9 years). The mortality density for patients with $\mathrm{N} 2 / \mathrm{N} 3$ of lymph node metastasis and moderate/poor tumor differentiation was 23.5 and 9.0 per 100 people-years, respectively. A higher mortality risk was observed to be related to a higher level of tumor size, lymph node metastasis, tumor stage, tumor differentiation and chemotherapy/radiotherapy $(\mathrm{aHR}=2.0,3.0,2.7,2.9$ and 3.7, respectively). The independent mortality risk for patients with high ANP32A expression was non-significant as compared with those with low expression, regardless the use of Allred and IRS scoring systems (both $P>0.05$ ).

Because ANP32A expression was associated with lymph node metastasis and tumor differentiation, we further evaluated the joint effect of ANP32A expression and the two pathological factors on mortality. The combined Kaplan-Meier mortality curves associated with lymph node stage and ANP32A expression using Allred and IRS scoring systems were significantly heterogeneous $(P<0.001$ and 0.002 , respectively, Supplementary figure 1 and 2). Similar findings were found in the joint mortality curves in relation to tumor differentiation and ANP32A expression (Supplementary figure 3 and 4). As compared to patients with N0/N1 stage and low ANP32A expression, the mortality hazard risk was multiplicatively enhanced among patients with $\mathrm{N} 2 / \mathrm{N} 3$ stage and high ANP32A expression $(\mathrm{aHR}=1.8,95 \% \mathrm{CI}, 1.1-3.8 ; P=0.008$ for multiplicative interaction, Table 3).Focusing on OSCC patients with $\mathrm{N} 2 / \mathrm{N} 3$ stage of lymph node metastasis $(N$
= 59), patients with Allred defined high level of ANP32A expression was observed to have a worse survival curves $(P=0.049$ for log-rank tests, Figure 2$)$ and a 2.1-fold higher hazard risk (95\% CI, 1.0-4.2) than patients with low ANP32A expression.

\section{ANP32A protein expression in OSCC-derived cell lines}

To investigate ANP32A function, we examined ANP32A levels in a panel of human OSCC cell lines. As shown in Figure 3a, ANP32A was found to over express to varying degrees in oral cancer cells. A high metastatic human tongue cancer cell line HSC-3 that showed a high ANP32A expression was chosen to do the further experiments. To evaluate the significance of ANP32A in high metastatic cells, we transiently transfected siANP32A for $72 \mathrm{~h}$ and then analyzed for its mRNA and protein expression. The amount of ANP32A mRNA and protein levels was reduced greatly compared with the negative control cells (Figure $3 \mathrm{~b} \& 3 \mathrm{c}$ ). Our results showed that $48 \mathrm{~h}$ time duration was sufficient to block the mRNA and protein expression of ANP32A. Thus we chose $48 \mathrm{~h}$ time point to do the downstream experiment.

\section{Effect of ANP32A depletion on EMT}

Because the clinical significance of ANP32A overexpression was linked to lymphnode metastasis, we evaluated the expression difference of several epithelialmesenchymal transition (EMT) markers between ANP32A knockdown cells and control cells. We found a significant decrease in Slug, Claudin-1 and Vimentin while an 
Table 3: The effect of clinicopathologic factor and ANP32A expression on mortality density and adjusted hazard ratio (aHR) among OSCC patients

\begin{tabular}{|c|c|c|c|c|c|c|}
\hline Factors & \begin{tabular}{|l|} 
No. of \\
patient
\end{tabular} & \begin{tabular}{|l} 
Follow-up \\
(person-year)
\end{tabular} & \begin{tabular}{|l|}
$\begin{array}{l}\text { No. of } \\
\text { death }\end{array}$ \\
\end{tabular} & $\begin{array}{l}\text { Mortality } \\
\text { density }^{\text {a }}\end{array}$ & $\mathbf{a H R}^{\mathbf{b}}$ & $(95 \% \mathrm{CI})$ \\
\hline \multicolumn{7}{|c|}{ Overall mortality from primary malignancy to death } \\
\hline \multicolumn{7}{|l|}{ T classification } \\
\hline $\mathrm{I} / \mathrm{II}$ & 145 & 890.9 & 46 & 5.2 & 1.0 & \\
\hline III/IV & 114 & 496.4 & 61 & 12.3 & 2.0 & $(1.4-3.0)$ \\
\hline \multicolumn{7}{|l|}{$\mathrm{N}$ classification } \\
\hline $\mathrm{N} 0 / \mathrm{N} 1$ & 200 & 1221.4 & 68 & 5.6 & 1.0 & \\
\hline $\mathrm{N} 2 / \mathrm{N} 3$ & 59 & 165.8 & 39 & 23.5 & 3.0 & $(2.0-4.5)$ \\
\hline \multicolumn{7}{|l|}{ AJCC tumor stage } \\
\hline $\mathrm{I} / \mathrm{II}$ & 104 & 717.6 & 26 & 3.6 & 1.0 & \\
\hline III/IV & 155 & 669.7 & 81 & 12.1 & 2.7 & $(1.7-4.3)$ \\
\hline \multicolumn{7}{|l|}{ Tumor differentiation } \\
\hline Well & 41 & 283.5 & 8 & 2.8 & 1.0 & \\
\hline Moderate/Poor & 218 & 1103.8 & 99 & 9.0 & 2.9 & $(1.4-6.1)$ \\
\hline \multicolumn{7}{|l|}{ Clinical therapy } \\
\hline Surgery & 98 & 690.1 & 20 & 2.9 & 1.0 & \\
\hline Chemotherapy/Radiotherapy & 161 & 697.1 & 87 & 12.5 & 3.7 & $(2.2-6.0)$ \\
\hline \multicolumn{7}{|l|}{ Allred } \\
\hline Low & 151 & 816.8 & 59 & 7.2 & 1.0 & \\
\hline High & 108 & 570.4 & 48 & 8.4 & 1.2 & $(0.8-1.8)$ \\
\hline \multicolumn{7}{|l|}{ IRS } \\
\hline Low & 159 & 870.1 & 61 & 7.0 & 1.0 & \\
\hline High & 100 & 517.1 & 46 & 8.9 & 1.3 & $(0.9-1.9)$ \\
\hline \multicolumn{7}{|l|}{ Combined effect } \\
\hline \multicolumn{7}{|l|}{$\mathrm{N}$ stage/Allred ${ }^{\mathrm{c}}$} \\
\hline N0+N1/Low & 125 & 732.4 & 46 & 6.3 & 1.0 & \\
\hline N0+N1//High & 75 & 489.0 & 22 & 4.5 & 0.6 & $(0.4-1.1)$ \\
\hline N2+N3/Low & 26 & 84.4 & 13 & 15.4 & 0.9 & $(0.5-1.8)$ \\
\hline N2+N3/High & 33 & 81.4 & 26 & 32.0 & 1.8 & $(1.1-3.8)^{\mathrm{d}}$ \\
\hline \multicolumn{7}{|l|}{ N stage/IRS ${ }^{c}$} \\
\hline N0+N1/Low & 130 & 783.4 & 45 & 5.7 & 1.0 & \\
\hline N0+N1//High & 70 & 438.0 & 23 & 5.3 & 0.8 & $(0.4-1.3)$ \\
\hline N2+N3/Low & 29 & 86.7 & 16 & 18.5 & 1.2 & $(0.6-2.2)$ \\
\hline N2+N3/High & 30 & 79.1 & 23 & 29.1 & 1.8 & $(1.0-3.1)$ \\
\hline \multicolumn{7}{|c|}{ Overall mortality from malignancy with $\mathrm{N} 2+\mathrm{N} 3$ stage to death } \\
\hline \multicolumn{7}{|l|}{ Allred $^{\mathrm{c}}$} \\
\hline Low & 26 & 84.4 & 13 & 15.4 & 1.0 & \\
\hline High & 33 & 81.4 & 26 & 32.0 & 2.1 & $(1.0-4.2)$ \\
\hline \multicolumn{7}{|l|}{$\mathrm{IRS}^{\mathrm{c}}$} \\
\hline Low & 29 & 86.7 & 16 & 18.5 & 1.0 & \\
\hline High & 30 & 79.1 & 23 & 29.1 & 1.6 & $(0.8-3.1)$ \\
\hline
\end{tabular}

${ }^{a}$ Mortaility density was displayed as per 100 people-years.

baHR was adjusted for gender and age.

${ }^{c} \mathrm{HR}$ was adjusted for gender, age, tumor differentiation, tumor stage and therapy method.

${ }^{\mathrm{d}}$ Significant multiplicative-scale interaction between N2+N3 stage and high expression on mortality risk was identified $(P$ for interaction, 0.008). 


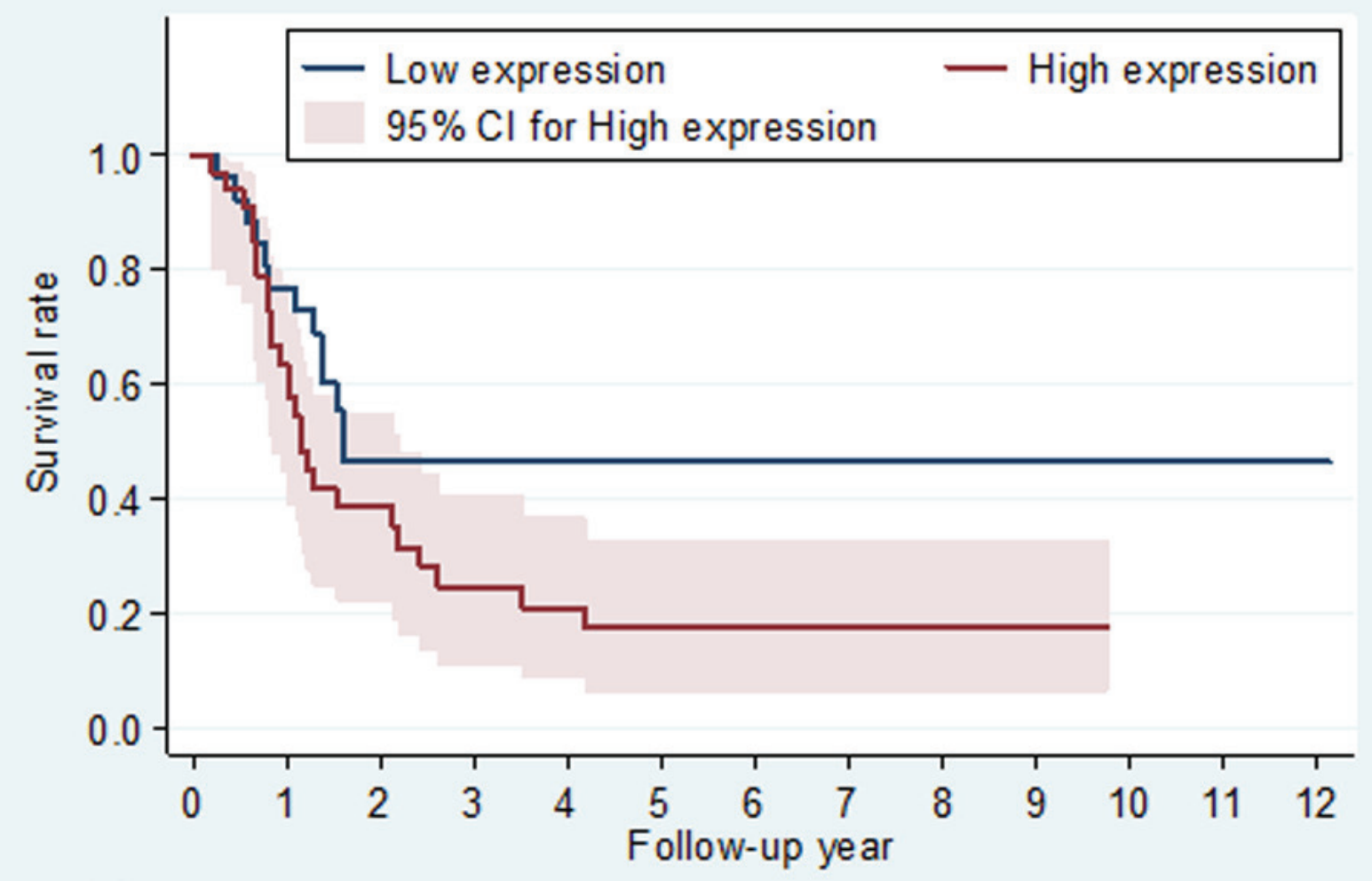

Number at risk

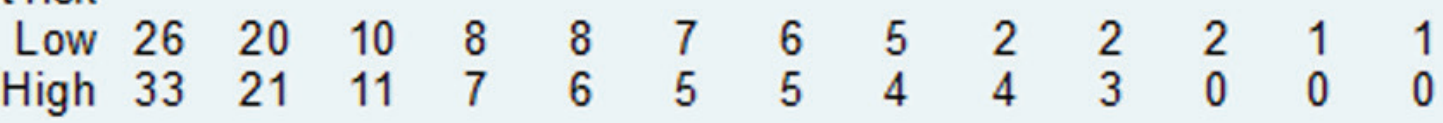

Figure 2: Kaplan-Meier survival curves and at-risk tables associated with ANP32A expression defined by the Allred scoring system among oral cancer patients with $\mathbf{N 2}+\mathbf{N} 3$ lymph node stage. $P$-values obtained from log-rank tests for the homogeneity of Kaplan-Meier curves between high and low ANP32A expressions was 0.049 .

a)

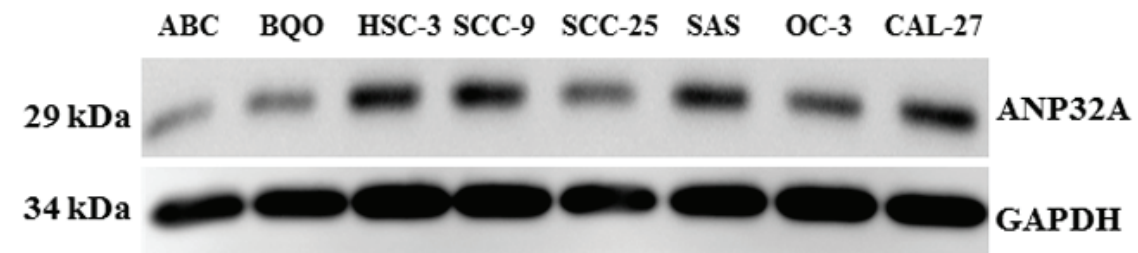

b)

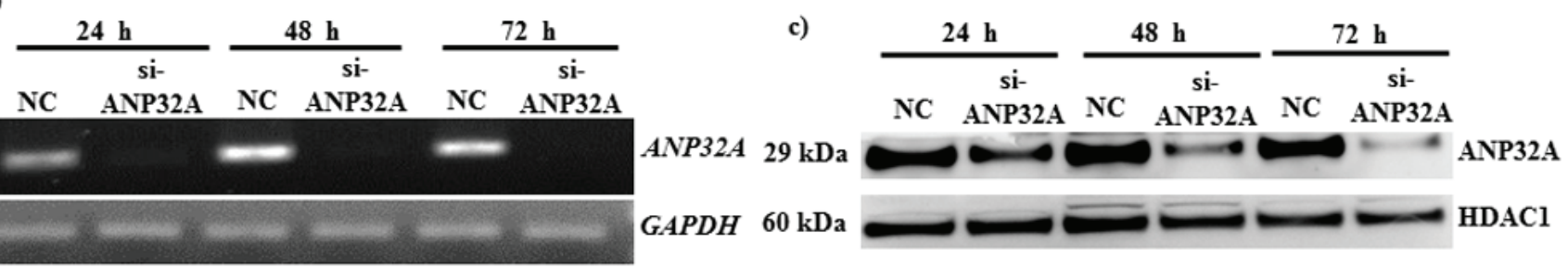

Figure 3: Immunoblot analysis of ANP32A expression. a. Oral cancer cells were analyzed for ANP32A expression; GAPDH was used as a loading control. HSC-3 cells were transfected with siControl (siCtrl) or si-ANP32A for different time points and analyzed for ANP32A b. mRNA expression by RT-PCR, and c) protein expression by western blotting. GAPDH and HDAC1 were used as an internal control, respectively. 
epithelial marker E-cadherin was increased in HSC-3 (Figure 4).

\section{Reduced ANP32A expression decreases OSCC cell migration and invasion}

HSC-3 cells were treated with si-ANP32A for $48 \mathrm{~h}$ and then seeded in Boyden chamber with or without matrigel for $24 \mathrm{~h}$ to determine whether ANP32A blockade could decrease their migration and invasion potential. ANP32A inhibition dramatically decreased the migration ability and number of migrated cells when compared to the negative control group (Figure 5a). We further examined the invasive ability in ANP32A inhibited group; ANP32A inhibition remarkably decreased the number of invaded cells compared with the negative control (Figure 5b). These findings suggest ANP32A expression may involve in EMT progression to promote invasion and metastasis of HSC-3.

\section{DISCUSSION}

It has been long recognized that ANP32A function as a tumor suppressor in several human cancers, including pancreatic $[9,13,17]$ and breast cancer $[19,20]$. In Pancreatic cancer, their expression level was highly associated with tumor differentiation [17]. However, no any information is available about ANP32A expression and its prognostic significance in OSCC. In this present study we identified and quantified ANP32A protein expression in OSCC tissue and normal marginal tissues by IHC. We found that nucleus expression of ANP32A was abundantly found in all the samples $(100 \%)$ and positive cytoplasmic expression was found in $73.7 \%$ of patients. Our findings also showed that high ANP32A protein level was associated with a higher level of lymph node metastasis and tumor differentiation, but not with TM classification and tumor stage.

The presence of lymph node metastasis at the time of diagnosis is one of the most important prognostic factors in OSCC[21].Therefore, an efficient biomarker is essential for earlier determination of lymph node metastasis that may help physician to have their therapy options [22]. In this present study, we found that lymph node metastasis (N2/N3) patients with high ANP32A expression had a worst prognosis at a 10 year follow up. To our knowledge, this is the first study that shows the correlation between ANP32A expression by IHC and lymph node metastasis and overall survival among OSCC patients using Allred and IRS system. However, no any significant association was found between ANP32A expression and clinical characteristics. From our study it is evident that, Allred and IRS can be used as a golden standard system to evaluate ANP32A expression in various other cancers. Taken together, these results indicate that ANP32A could
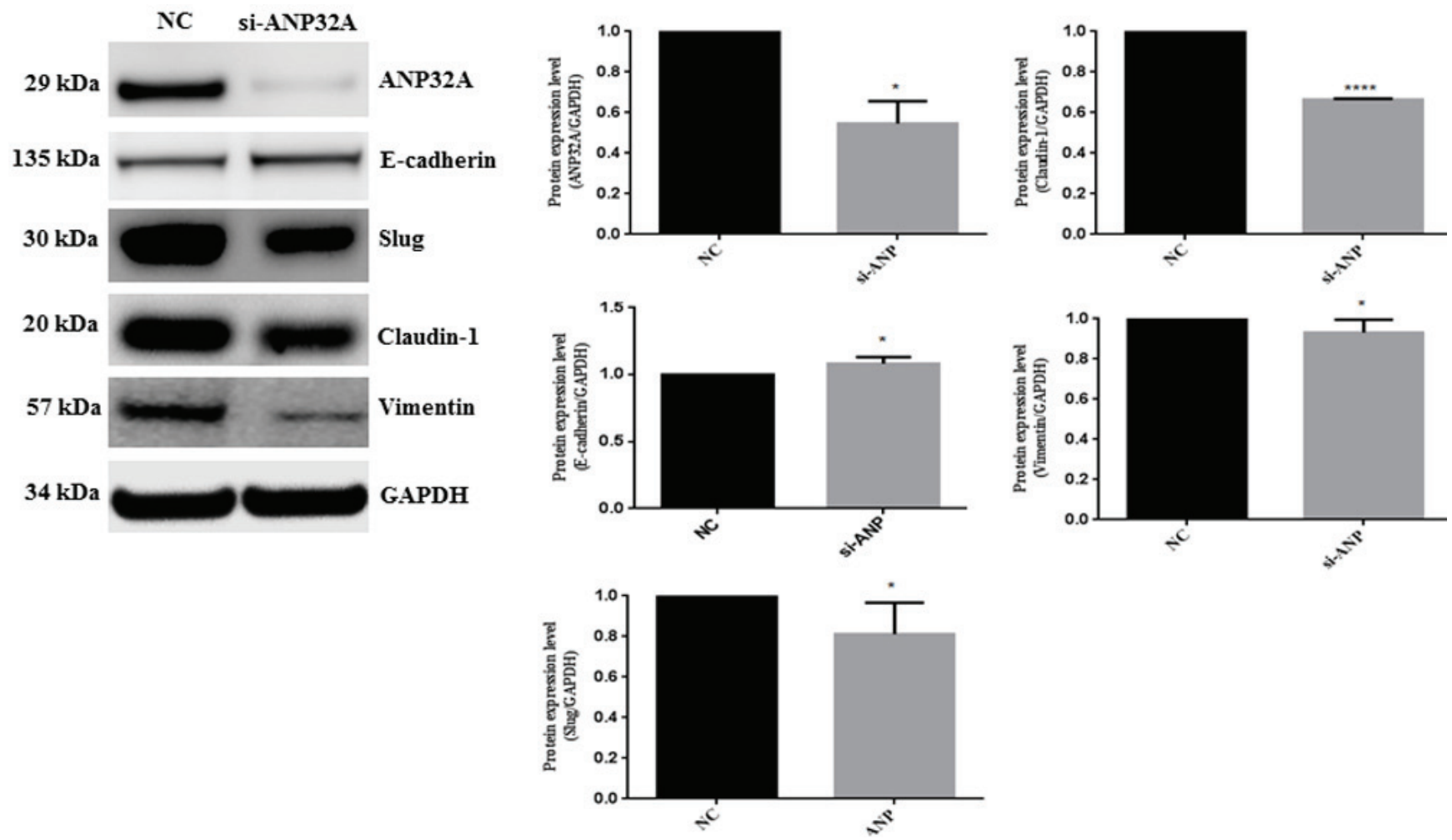

Figure 4: Knock down of endogenous ANP32A expression altered EMT proteins- E-cadherin, Slug, Claudlin-1 and Vimentin expression. GAPDH was used as a loading control. Densitometry values are represented in bar chart. Data mean values \pm $\mathrm{SD}$. The asterisks indicate a significant $(*, P<0.05, * * P<0.01)$ difference between the si Control (siCtrl) and si-ANP32A treated groups. 
serve as a potential biomarker of lymph node metastasis in OSCC.

Earlier studies suggested a role for ANP32A expression as a prognostic biomarker in pancreatic cancer. Brody et al correlated ANP32A expression in well-differentiated adenocarcinomas, and in a subset of moderately differentiated adenocarcinomas. ANP32A was absent or reduced in poorly differentiated tumors and in intraductal papillary mucinous neoplasms with moderate dysplasia [13]. However, it has been reported that ANP32A overexpression was not significantly correlated with pathological parameter in cancers like pancreatic and non-small cell lung cancer. Thus, ANP32A expression seems to behave in a tissue specific manner.

In this present study, ANP32A expression was significantly associated with oral cancer tumor invasiveness and lymphnode metastasis. Therefore, ANP32A-expressing cells have clinical implication for the development and progression of OSCC. The level of ANP32A expression may be considered to use as a biomarker for malignancy of OSCC and a predictor of patient prognosis. We further investigated whether ANP32A over expression increased the invasive potential of oral carcinoma cell lines. A highly malignant and invasive oral cancer HSC-3cell isolated from metastatic tumors in a lymph node was chosen to perform the in vitro study [23, 24]. Abrogating ANP32A expression in highly invasive oral cancer cell decreases its metastasis and invasive levels. Our findings suggest that ANP32A expression increases as cancer cells progress toward malignant phenotype. EMT is critical in tumor progression and metastasis formation and it is related with worse prognosis for cancer patients [25, 26]. During EMT transformation epithelial markers (such as E-cadherin, desmoplakin, cytokeratins, claudins, occluding, and betacatenin) were decreased and the mesenchymal markers (such as vimentin, n-cadherin, fibronectin, and snail-1/2) were increased [27-30]. Recent studies have shown that claudin-1 is aberrantly expressed in diverse types of human cancers, including OSCCs [31]. In this study, we found that knockdown of ANP32A decreased Snail, Vimentin, Claudin-1 and increased the level of E-cadherin expression in vitro. We found highly significant association between ANP32A and Claudin-1expression compared with other EMT marker. These results support the notion that ANP32A increased cancer cell invasion through EMT
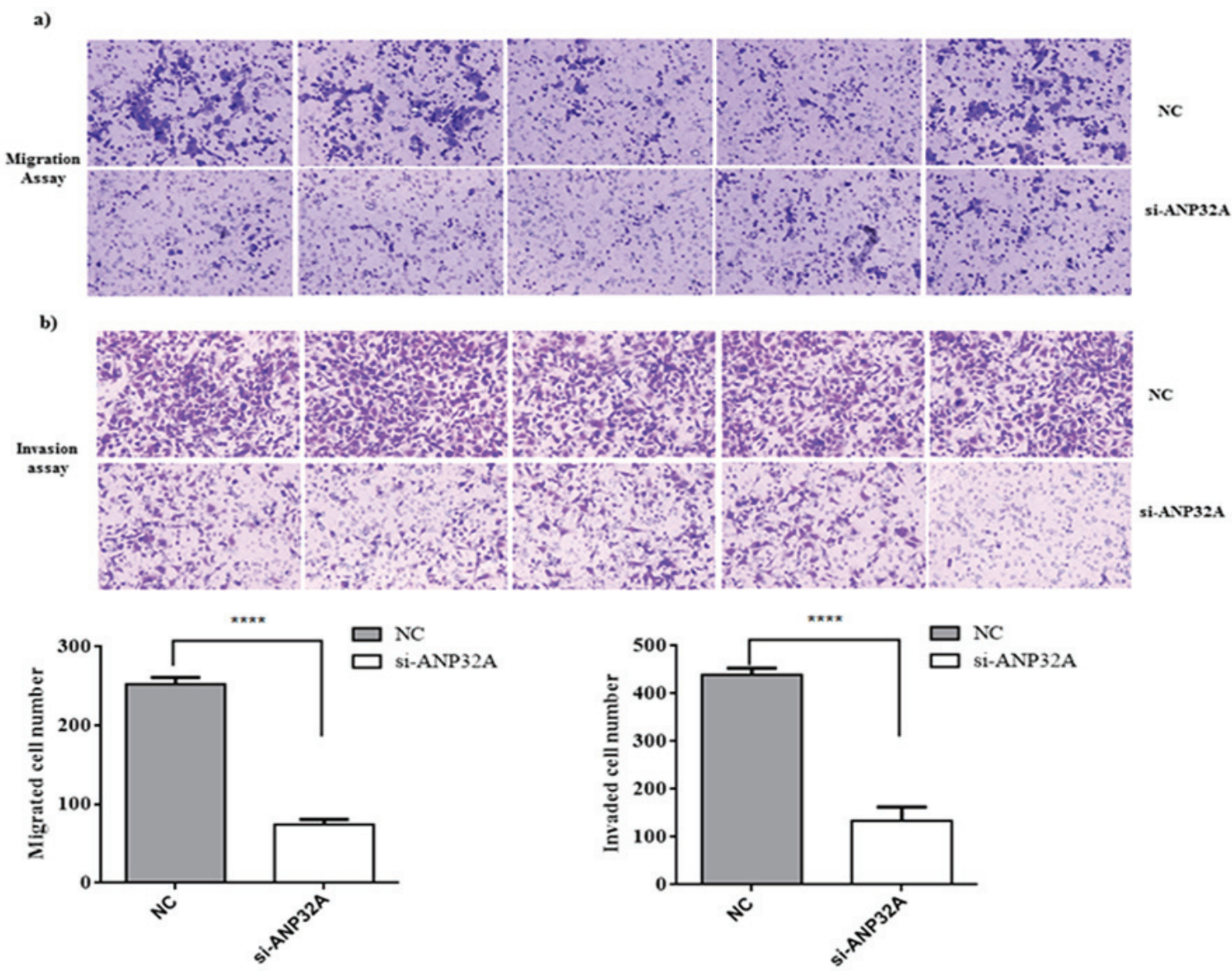

Figure 5: Knockdown of ANP32A reduces OSCC cell migration and invasion. HSC-3 cells were transiently transfected with ANP32A-specific siRNA, a nonspecific siRNA (negative control). Representative photos of a. migration assay and b.matrigel invasion assay using Boyden chamber. Quantification of relative numbers of migrated and invaded cells represents average counts from five fields of view. Data mean values \pm SD. $* * * * P<0.0001$. 
pathway.

In conclusion we report here for the first time, that ANP32A is aberrantly expressed in OSCC tissues and in OSCC-derived cell lines. High ANP32A expression was associated with high stage of lymphnode metastasis at cancer diagnosis, and the mortality hazard risk was multiplicatively enhanced among N2/N3 patients with high ANP32A expression. Our in vitro results further showed that, knockdown of ANP32A in oral cancer cells reduced its invasive and metastatic ability. However, further investigations are necessary to understand their molecular role in oral cancer progression and metastasis.

\section{MATERIALS AND METHODS}

\section{Participants and clinical tissues}

To evaluate the relationship of ANP32A expression with clinical/pathological factors and survival, a total of 259 oral cancer tissue samples from tissue blocks were obtained from the Changhua Christian Hospital. The tumors were classified according to the International Union against Cancer TNM classification system [32]. Only specimens without any treatment were selected for this study to avoid possible influences of the treatment modality. The present study was approved by the ethics committee of China medical University Hospital and Changhua Christian Hospital according to the declaration of Helsinki. Informed consent was obtained from all patients for this study.

\section{OSCC cell lines}

$\mathrm{ABC}$ and $\mathrm{BQO}$ primary oral cancer cells were originally established in our lab. OC-3 cell were obtained from Dr. S.C. Lin (National Yang Ming University, Taipei, Taiwan)[33] and oral cancer cell lines, SAS, HSC-3, SCC9, SCC-25, SAS and CAL-27 were purchased from the Bio-resource Collection and Research Center, Taiwan and maintained in DMEM- F12 (Invitrogen; Carlsbad, CA) medium supplemented with $10 \%$ fetal bovine serum. OC-3 cells were cultured in a 1:2 mixture of DMEM-F12 and KSFM with $10 \%$ FBS. All the cells are incubated in a humidified $5 \% \mathrm{CO}_{2}$ atmosphere at $37^{\circ} \mathrm{C}$.

\section{Tissue microarray}

Formalin-fixed, paraffin-embedded block of cancer tissues are used in this experiment. The tissue cores were arranged into new paraffin blocks by using a fine steel needle to create the tissue microarrays. Cancer tissues were sliced to $4 \mu \mathrm{m}$ thickness and then hematoxylin and eosin staining was performed to confirm the presence of the original cancers as indicated by morphologically representative areas [34].

\section{Immunohistochemical staining and scoring}

The Immunohistochemical staining methods have been described previously[34]. Tissue sections were deparaffinized and rehydrated using routine techniques. Endogenous peroxidase activity was blocked with 3\% $\mathrm{H}_{2} \mathrm{O}_{2}$ in methanol, hydrated with gradient alcohol and phosphate-buffered saline solution, and incubated in $10 \mathrm{mmol} / \mathrm{L}$ citrate buffer ( $\mathrm{pH}$ 6.0). The sections were incubated with ANP32A rabbit polyclonal antibody (Abcam, Cambridge, United Kingdom) in room temperature for 20 mins. After washing three times with PBS, the sections were incubated with appropriate peroxidase-labelled secondary antibodies for $30 \mathrm{~min}$ at room temperature. The sections were washed three times with PBS and then labelled by diaminobenzidine and counterstained with Mayer's haematoxylin, dehydrated and mounted.

Each tumor was given a score according to the intensity of the nuclear/cytoplasmic staining: Negative staining and weak positive staining, \pm ; moderate staining, $1+$; and strong staining, 2+. Staining intensity was confirmed by 2 pathologists. For Allred scoring system, the cut-off score $\leq 5$ and $>5$ were defined as low and high expression level, respectively. For Klein scoring system and immunoreactive scoring (IRS) system, the cut-off score $\leq 3$ and $>3$ were defined as low and high expression level, respectively.

\section{Small interfering RNA transfections}

Double-stranded small interfering RNA (siRNA) oligonucleotides $(25$ pmol per $6 \mathrm{~cm}$ plate; ANP32A: 5'-UUUGGUAAGUUUGCGAUUGag-3'; CY3 dye labeled pre-miR Negative Control 1 (Ambion, Foster City, CA, AM17120), both were transfected with RNAiMAX reagent (Invitrogen, Carlsbad, CA) according to the manufacturer's instructions.

\section{Migration and invasion assay}

Invasion assay was performed as mentioned earlier [35]. Cell migration and invasion was performed at a 24well Transwell chamber with a pore size of $8 \mu \mathrm{m}$ (Corning, Bedford, MA, USA). The insert was coated with $100 \mu \mathrm{l}$ Matrigel (dilution at 1:2; Corning, Bedford, MA, USA). HSC-3 cells were trypsinised after transfection with control or ANP32A siRNA for $48 \mathrm{~h}$ and transferred to the upper chamber with or without Matrigel in $100 \mu$ l of serum free medium containing cells and incubated for $24 \mathrm{~h}$. The lower chamber was filled with complete media. 
The membranes were fixed and stained using $0.1 \%$ crystal violet.

\section{Western blot analysis}

Immunoblotting was performed with primary antibodies against ANP32A (Abcam, Cambridge, United Kingdom), E-cadherin, Slug, Vimentin and Claudin-1 (Cell Signaling Technology, Danvers, MA, USA). GAPDH (Millipore, Billerica, MA, USA), HDAC1 (Santa Cruz Biotechnology, Santa Cruz, CA, USA) was used as a loading control. The signal was visualized with super enhanced chemiluminescence (ECL) detection reagent (Millipore, Billerica, MA, USA).

\section{RNA isolation and real-time PCR}

Total RNA was isolated from HSC-3 cells transfected with negative control or ANP32A siRNAs, using the Trizol reagent (Invitrogen, Burlington, ON, USA) according to the manufacturer's instruction. Isolated RNA was used as a template for the reverse transcription reaction (Invitrogen, Carlsbad, CA). The ANP32A sense primer was 5'-GCCTCACCTCAATCGCAAAC-3', and the antisense primer was 5'- CCCCTGAGACTCTGTTATCGC-3'. For GAPDH gene, the sense primer was 5'GAGAAGGCTGGGGCTCATTT-3' and the antisense primer was 5' - AGTGATGGCATGGACTGTGG-3'.

\section{Statistical analysis}

Proportions were used to describe the distribution of demographic and clinicopathologic characteristics in the patient cohort with oral squamous cell carcinoma. Multivariate logistic regression models were employed to evaluate the association between clinicopathologic factors and ANP32A expression defined by Allred, Klein and IRS scoring systems, respectively. We used Kaplan-Meier curves to assess survival of the patients having diverse ANP32A expression over time, with differences appraised using the log-rank test for equality of survivor functions. The adjusted hazard ratio (aHR) and corresponding 95\% confidence intervals (CIs) obtained from the multivariate Cox proportional-hazards regression models were employed to determine the predictive effect of ANP32A expression on survival. Overall survival was separately computed for all patients and for the patients with N2 or N3 lymph node stages. The two overall survivals were calculated from the date of primary malignancy diagnosis and the date of diagnosis of N2 or N3 lymph node stages to the last follow-up (censored) or death from malignancy (event), respectively. Gender and age, and where appropriate, tumor stage, tumor differentiation and therapy scheme were included in the multivariate Cox model as the covariates. All $P$ values were two-tailed, and a $P$ value of $<0.05$ was considered statistically significant.

\section{ACKNOWLEDGMENTS}

The study was supported by grants from the Ministry of Science and Technology MOST103-2314-B-039-003 and MOST104-2314-B-039-003, Ministry of Health and Welfare MOHW 104-TDU-B-212-124-002, and Experiments and data analysis were performed in part through the use of the Medical Research Core Facilities Center, office of Research \& Development at China Medical University, Taichung, Taiwan.

\section{CONFLICTS OF INTEREST}

The authors declare no conflict of interest.

\section{REFERENCES}

1. Ko YC, Huang YL, Lee CH, Chen MJ, Lin LM and Tsai CC. Betel quid chewing, cigarette smoking and alcohol consumption related to oral cancer in Taiwan. Journal of oral pathology \& medicine : official publication of the International Association of Oral Pathologists and the American Academy of Oral Pathology. 1995; 24:450-453.

2. Ross GL, Soutar DS, Gordon MacDonald D, Shoaib T, Camilleri I, Roberton AG, Sorensen JA, Thomsen J, Grupe P, Alvarez J, Barbier L, Santamaria J, Poli T, Massarelli O, Sesenna E, Kovacs AF, et al. Sentinel node biopsy in head and neck cancer: preliminary results of a multicenter trial. Ann Surg Oncol. 2004; 11:690-696.

3. Gupta S, Kong W, Peng Y, Miao Q and Mackillop WJ. Temporal trends in the incidence and survival of cancers of the upper aerodigestive tract in Ontario and the United States. International journal of cancer. 2009; 125:21592165.

4. Silverman S, Jr. Demographics and occurrence of oral and pharyngeal cancers. The outcomes, the trends, the challenge. J Am Dent Assoc. 2001; 132 Suppl:7S-11S.

5. Yoshida R, Nakayama H, Nagata M, Hirosue A, Tanaka T, Kawahara K, Nakagawa Y, Matsuoka Y, Sakata J, Arita H, Hiraki A, Shinohara M and Ito T. Overexpression of nucleostemin contributes to an advanced malignant phenotype and a poor prognosis in oral squamous cell carcinoma. British journal of cancer. 2014; 111:2308-2315.

6. Lai MT, Hua CH, Tsai MH, Wan L, Lin YJ, Chen CM, Chiu IW, Chan C, Tsai FJ and Jinn-Chyuan Sheu J. Talin-1 overexpression defines high risk for aggressive oral squamous cell carcinoma and promotes cancer metastasis. J Pathol. 2011; 224:367-376.

7. Kadkol SS, Brody JR, Epstein JI, Kuhajda FP and Pasternack GR. Novel nuclear phosphoprotein pp32 is highly expressed in intermediate- and high-grade prostate cancer. The Prostate. 1998; 34:231-237. 
8. Adegbola $\mathrm{O}$ and Pasternack GR. Phosphorylated retinoblastoma protein complexes with pp32 and inhibits pp32-mediated apoptosis. The Journal of biological chemistry. 2005; 280:15497-15502.

9. Bai J, Brody JR, Kadkol SS and Pasternack GR. Tumor suppression and potentiation by manipulation of pp32 expression. Oncogene. 2001; 20:2153-2160.

10. Theodosiou A and Ashworth A. MAP kinase phosphatases. Genome biology. 2002; 3:REVIEWS3009.

11. Millward TA, Zolnierowicz $\mathrm{S}$ and Hemmings BA. Regulation of protein kinase cascades by protein phosphatase 2A. Trends in biochemical sciences. 1999; 24(5):186-191.

12. Chakravarti D and Hong R. SET-ting the stage for life and death. Cell. 2003; 112:589-591.

13. Brody JR, Witkiewicz A, Williams TK, Kadkol SS, Cozzitorto J, Durkan B, Pasternack GR and Yeo CJ. Reduction of pp32 expression in poorly differentiated pancreatic ductal adenocarcinomas and intraductal papillary mucinous neoplasms with moderate dysplasia. Mod Pathol. 2007; 20:1238-1244.

14. Schafer ZT, Parrish AB, Wright KM, Margolis SS, Marks JR, Deshmukh M and Kornbluth S. Enhanced sensitivity to cytochrome c-induced apoptosis mediated by PHAPI in breast cancer cells. Cancer research. 2006; 66:2210-2218.

15. Li C, Ruan HQ, Liu YS, Xu MJ, Dai J, Sheng QH, Tan YX, Yao ZZ, Wang HY, Wu JR and Zeng R. Quantitative proteomics reveal up-regulated protein expression of the SET complex associated with hepatocellular carcinoma. Journal of proteome research. 2012; 11:871-885.

16. Shi H, Hood KA, Hayes MT and Stubbs RS. Proteomic analysis of advanced colorectal cancer by laser capture microdissection and two-dimensional difference gel electrophoresis. Journal of proteomics. 2011; 75:339-351.

17. Williams TK, Costantino CL, Bildzukewicz NA, Richards NG, Rittenhouse DW, Einstein L, Cozzitorto JA, Keen JC, Dasgupta A, Gorospe M, Gonye GE, Yeo CJ, Witkiewicz AK and Brody JR. pp32 (ANP32A) expression inhibits pancreatic cancer cell growth and induces gemcitabine resistance by disrupting HuR binding to mRNAs. PloS one. 2010; 5:e15455.

18. Fedchenko N and Reifenrath J. Different approaches for interpretation and reporting of immunohistochemistry analysis results in the bone tissue - a review. Diagnostic pathology. 2014; 9:221.

19. Kadkol SS, El Naga GA, Brody JR, Bai J, Gusev Y, Dooley WC and Pasternack GR. Expression of pp32 gene family members in breast cancer. Breast Cancer Res Treat. 2001; 68:65-73.

20. Zamora-Caballero S, Siauciunaite-Gaubard L and Bravo J. High-resolution crystal structure of the leucine-rich repeat domain of the human tumour suppressor PP32A (ANP32A). Acta Crystallogr F Struct Biol Commun. 2015; 71:684-687.

21. Kim SY, Nam SY, Choi SH, Cho KJ and Roh JL.
Prognostic value of lymph node density in node-positive patients with oral squamous cell carcinoma. Ann Surg Oncol. 2011; 18:2310-2317.

22. Noorlag R, van der Groep P, Leusink FKJ, van Hooff SR, Frank MH, Willems SM and van Es RJJ. Nodal metastasis and survival in oral cancer: Association with protein expression of SLPI, not with LCN2, TACSTD2, or THBS2. Head Neck-J Sci Spec. 2015; 37:1130-1136.

23. Rikimaru K, Toda H, Tachikawa N, Kamata N and Enomoto S. Growth of the malignant and nonmalignant human squamous cells in a protein-free defined medium. In Vitro Cell Dev Biol. 1990; 26:849-856.

24. Wu MH, Hong TM, Cheng HW, Pan SH, Liang YR, Hong HC, Chiang WF, Wong TY, Shieh DB, Shiau AL, Jin YT and Chen YL. Galectin-1-mediated tumor invasion and metastasis, up-regulated matrix metalloproteinase expression, and reorganized actin cytoskeletons. Mol Cancer Res. 2009; 7:311-318.

25. Kreizenbeck GM, Berger AJ, Subtil A, Rimm DL and Gould Rothberg BE. Prognostic significance of cadherinbased adhesion molecules in cutaneous malignant melanoma. Cancer Epidemiol Biomarkers Prev. 2008; 17:949-958.

26. Christiansen JJ and Rajasekaran AK. Reassessing epithelial to mesenchymal transition as a prerequisite for carcinoma invasion and metastasis. Cancer research. 2006; 66:83198326.

27. Zhu LF, Hu Y, Yang CC, Xu XH, Ning TY, Wang ZL, Ye $\mathrm{JH}$ and Liu LK. Snail overexpression induces an epithelial to mesenchymal transition and cancer stem cell-like properties in SCC9 cells. Lab Invest. 2012; 92:744-752.

28. Cavallaro U and Christofori $G$. Cell adhesion and signalling by cadherins and Ig-CAMs in cancer. Nat Rev Cancer. 2004; 4:118-132.

29. Lin TC, Liu YP, Chan YC, Su CY, Lin YF, Hsu SL, Yang $\mathrm{CS}$ and Hsiao M. Ghrelin promotes renal cell carcinoma metastasis via Snail activation and is associated with poor prognosis. J Pathol. 2015; 237:50-61.

30. Thiery JP. Epithelial-mesenchymal transitions in tumour progression. Nature Reviews Cancer. 2002; 2:442-454.

31. Dos Reis PP, Bharadwaj RR, Machado J, Macmillan C, Pintilie M, Sukhai MA, Perez-Ordonez B, Gullane P, Irish $\mathrm{J}$ and Kamel-Reid S. Claudin 1 overexpression increases invasion and is associated with aggressive histological features in oral squamous cell carcinoma. Cancer. 2008; 113:3169-3180.

32. Kreppel M, Eich HT, Kubler A, Zoller JE and Scheer M. Prognostic value of the sixth edition of the UICC's TNM classification and stage grouping for oral cancer. Journal of surgical oncology. 2010; 102:443-449.

33. Chiang SL, Chen PH, Lee $\mathrm{CH}$, Ko AM, Lee KW, Lin YC, Ho PS, Tu HP, Wu DC, Shieh TY and Ko YC. Upregulation of inflammatory signalings by areca nut extract and role of cyclooxygenase-2 $-1195 \mathrm{G}>$ a polymorphism 
reveal risk of oral cancer. Cancer research. 2008; 68:84898498.

34. Hsu LS, Wu PR, Yeh KT, Yeh CM, Shen KH, Chen CJ and Soon MS. Positive nuclear expression of KLF8 might be correlated with shorter survival in gastric adenocarcinoma. Ann Diagn Pathol. 2014; 18:74-77.

35. Ren J, Li W, Yan L, Jiao W, Tian S, Li D, Tang Y, Gu $\mathrm{G}$, Liu $\mathrm{H}$ and $\mathrm{Xu} \mathrm{Z}$. Expression of CIP2A in renal cell carcinomas correlates with tumour invasion, metastasis and patients' survival. British journal of cancer. 2011; 105:1905-1911. 